Ann. Funct. Anal. 7 (2016), no. 4, 646-655

http://dx.doi.org/10.1215/20088752-3660990

ISSN: 2008-8752 (electronic)

ANNALS of

FUNCTIONAL

http://projecteuclid.org/afa

ANALYSIS

\title{
DOMINATED OPERATORS FROM LATTICE-NORMED SPACES TO SEQUENCE BANACH LATTICES
}

\author{
NARIMAN ABASOV,${ }^{1}$ ABD EL MONEM MEGAHED,${ }^{2}$ and MARAT PLIEV ${ }^{3 *}$ \\ Communicated by M. de Jeu
}

\begin{abstract}
We show that every dominated linear operator from a BanachKantorovich space over an atomless Dedekind-complete vector lattice to a sequence Banach lattice $\ell_{p}(\Gamma)$ or $c_{0}(\Gamma)$ is narrow. As a consequence, we obtain that an atomless Banach lattice cannot have a finite-dimensional decomposition of a certain kind. Finally, we show that the order-narrowness of a linear dominated operator $T$ from a lattice-normed space $V$ to the Banach space with a mixed norm $(W, F)$ over an order-continuous Banach lattice $F$ implies the order-narrowness of its exact dominant $|T|$.
\end{abstract}

\section{INTRODUCTION AND PRELIMINARIES}

Narrow operators generalize compact operators defined on function spaces (see [11] for the first systematic study; see also the recent monograph [12]). Different classes of narrow operators in framework of vector lattices and latticenormed spaces were considered in [9], [10]. In the present article, we continue the investigation of narrow operators in lattice-normed spaces and show that every dominated linear operator from a Banach-Kantorovich space over an atomless Dedekind-complete vector lattice to a sequence Banach lattice is narrow. As a consequence, we obtain that an atomless Banach lattice cannot have a finitedimensional decomposition of a certain kind.

We also consider a domination problem for the exact dominant of a dominated linear operator. In the classical sense, the domination problem can be stated as

Copyright 2016 by the Tusi Mathematical Research Group.

Received Nov. 8, 2015; Accepted May 11, 2016.

${ }^{*}$ Corresponding author.

2010 Mathematics Subject Classification. Primary 46B42; Secondary $47 \mathrm{~B} 99$.

Keywords. narrow operators, dominated operators, lattice-normed spaces, Banach lattices. 
Ann. Funct. Anal. 7 (2016), no. 4, 646-655

http://dx.doi.org/10.1215/20088752-3660990

ISSN: 2008-8752 (electronic)

ANNALS of

FUNCTIONAL

http://projecteuclid.org/afa

ANALYSIS

\title{
DOMINATED OPERATORS FROM LATTICE-NORMED SPACES TO SEQUENCE BANACH LATTICES
}

\author{
NARIMAN ABASOV,${ }^{1}$ ABD EL MONEM MEGAHED,${ }^{2}$ and MARAT PLIEV ${ }^{3 *}$ \\ Communicated by M. de Jeu
}

\begin{abstract}
We show that every dominated linear operator from a BanachKantorovich space over an atomless Dedekind-complete vector lattice to a sequence Banach lattice $\ell_{p}(\Gamma)$ or $c_{0}(\Gamma)$ is narrow. As a consequence, we obtain that an atomless Banach lattice cannot have a finite-dimensional decomposition of a certain kind. Finally, we show that the order-narrowness of a linear dominated operator $T$ from a lattice-normed space $V$ to the Banach space with a mixed norm $(W, F)$ over an order-continuous Banach lattice $F$ implies the order-narrowness of its exact dominant $|T|$.
\end{abstract}

\section{INTRODUCTION AND PRELIMINARIES}

Narrow operators generalize compact operators defined on function spaces (see [11] for the first systematic study; see also the recent monograph [12]). Different classes of narrow operators in framework of vector lattices and latticenormed spaces were considered in [9], [10]. In the present article, we continue the investigation of narrow operators in lattice-normed spaces and show that every dominated linear operator from a Banach-Kantorovich space over an atomless Dedekind-complete vector lattice to a sequence Banach lattice is narrow. As a consequence, we obtain that an atomless Banach lattice cannot have a finitedimensional decomposition of a certain kind.

We also consider a domination problem for the exact dominant of a dominated linear operator. In the classical sense, the domination problem can be stated as

Copyright 2016 by the Tusi Mathematical Research Group.

Received Nov. 8, 2015; Accepted May 11, 2016.

${ }^{*}$ Corresponding author.

2010 Mathematics Subject Classification. Primary 46B42; Secondary $47 \mathrm{~B} 99$.

Keywords. narrow operators, dominated operators, lattice-normed spaces, Banach lattices. 
Ann. Funct. Anal. 7 (2016), no. 4, 646-655

http://dx.doi.org/10.1215/20088752-3660990

ISSN: 2008-8752 (electronic)

ANNALS of

FUNCTIONAL

http://projecteuclid.org/afa

ANALYSIS

\title{
DOMINATED OPERATORS FROM LATTICE-NORMED SPACES TO SEQUENCE BANACH LATTICES
}

\author{
NARIMAN ABASOV,${ }^{1}$ ABD EL MONEM MEGAHED,${ }^{2}$ and MARAT PLIEV ${ }^{3 *}$ \\ Communicated by M. de Jeu
}

\begin{abstract}
We show that every dominated linear operator from a BanachKantorovich space over an atomless Dedekind-complete vector lattice to a sequence Banach lattice $\ell_{p}(\Gamma)$ or $c_{0}(\Gamma)$ is narrow. As a consequence, we obtain that an atomless Banach lattice cannot have a finite-dimensional decomposition of a certain kind. Finally, we show that the order-narrowness of a linear dominated operator $T$ from a lattice-normed space $V$ to the Banach space with a mixed norm $(W, F)$ over an order-continuous Banach lattice $F$ implies the order-narrowness of its exact dominant $|T|$.
\end{abstract}

\section{INTRODUCTION AND PRELIMINARIES}

Narrow operators generalize compact operators defined on function spaces (see [11] for the first systematic study; see also the recent monograph [12]). Different classes of narrow operators in framework of vector lattices and latticenormed spaces were considered in [9], [10]. In the present article, we continue the investigation of narrow operators in lattice-normed spaces and show that every dominated linear operator from a Banach-Kantorovich space over an atomless Dedekind-complete vector lattice to a sequence Banach lattice is narrow. As a consequence, we obtain that an atomless Banach lattice cannot have a finitedimensional decomposition of a certain kind.

We also consider a domination problem for the exact dominant of a dominated linear operator. In the classical sense, the domination problem can be stated as

Copyright 2016 by the Tusi Mathematical Research Group.

Received Nov. 8, 2015; Accepted May 11, 2016.

${ }^{*}$ Corresponding author.

2010 Mathematics Subject Classification. Primary 46B42; Secondary $47 \mathrm{~B} 99$.

Keywords. narrow operators, dominated operators, lattice-normed spaces, Banach lattices. 
Ann. Funct. Anal. 7 (2016), no. 4, 646-655

http://dx.doi.org/10.1215/20088752-3660990

ISSN: 2008-8752 (electronic)

ANNALS of

FUNCTIONAL

http://projecteuclid.org/afa

ANALYSIS

\title{
DOMINATED OPERATORS FROM LATTICE-NORMED SPACES TO SEQUENCE BANACH LATTICES
}

\author{
NARIMAN ABASOV,${ }^{1}$ ABD EL MONEM MEGAHED,${ }^{2}$ and MARAT PLIEV ${ }^{3 *}$ \\ Communicated by M. de Jeu
}

\begin{abstract}
We show that every dominated linear operator from a BanachKantorovich space over an atomless Dedekind-complete vector lattice to a sequence Banach lattice $\ell_{p}(\Gamma)$ or $c_{0}(\Gamma)$ is narrow. As a consequence, we obtain that an atomless Banach lattice cannot have a finite-dimensional decomposition of a certain kind. Finally, we show that the order-narrowness of a linear dominated operator $T$ from a lattice-normed space $V$ to the Banach space with a mixed norm $(W, F)$ over an order-continuous Banach lattice $F$ implies the order-narrowness of its exact dominant $|T|$.
\end{abstract}

\section{INTRODUCTION AND PRELIMINARIES}

Narrow operators generalize compact operators defined on function spaces (see [11] for the first systematic study; see also the recent monograph [12]). Different classes of narrow operators in framework of vector lattices and latticenormed spaces were considered in [9], [10]. In the present article, we continue the investigation of narrow operators in lattice-normed spaces and show that every dominated linear operator from a Banach-Kantorovich space over an atomless Dedekind-complete vector lattice to a sequence Banach lattice is narrow. As a consequence, we obtain that an atomless Banach lattice cannot have a finitedimensional decomposition of a certain kind.

We also consider a domination problem for the exact dominant of a dominated linear operator. In the classical sense, the domination problem can be stated as

Copyright 2016 by the Tusi Mathematical Research Group.

Received Nov. 8, 2015; Accepted May 11, 2016.

${ }^{*}$ Corresponding author.

2010 Mathematics Subject Classification. Primary 46B42; Secondary $47 \mathrm{~B} 99$.

Keywords. narrow operators, dominated operators, lattice-normed spaces, Banach lattices. 
Ann. Funct. Anal. 7 (2016), no. 4, 646-655

http://dx.doi.org/10.1215/20088752-3660990

ISSN: 2008-8752 (electronic)

ANNALS of

FUNCTIONAL

http://projecteuclid.org/afa

ANALYSIS

\title{
DOMINATED OPERATORS FROM LATTICE-NORMED SPACES TO SEQUENCE BANACH LATTICES
}

\author{
NARIMAN ABASOV,${ }^{1}$ ABD EL MONEM MEGAHED,${ }^{2}$ and MARAT PLIEV ${ }^{3 *}$ \\ Communicated by M. de Jeu
}

\begin{abstract}
We show that every dominated linear operator from a BanachKantorovich space over an atomless Dedekind-complete vector lattice to a sequence Banach lattice $\ell_{p}(\Gamma)$ or $c_{0}(\Gamma)$ is narrow. As a consequence, we obtain that an atomless Banach lattice cannot have a finite-dimensional decomposition of a certain kind. Finally, we show that the order-narrowness of a linear dominated operator $T$ from a lattice-normed space $V$ to the Banach space with a mixed norm $(W, F)$ over an order-continuous Banach lattice $F$ implies the order-narrowness of its exact dominant $|T|$.
\end{abstract}

\section{INTRODUCTION AND PRELIMINARIES}

Narrow operators generalize compact operators defined on function spaces (see [11] for the first systematic study; see also the recent monograph [12]). Different classes of narrow operators in framework of vector lattices and latticenormed spaces were considered in [9], [10]. In the present article, we continue the investigation of narrow operators in lattice-normed spaces and show that every dominated linear operator from a Banach-Kantorovich space over an atomless Dedekind-complete vector lattice to a sequence Banach lattice is narrow. As a consequence, we obtain that an atomless Banach lattice cannot have a finitedimensional decomposition of a certain kind.

We also consider a domination problem for the exact dominant of a dominated linear operator. In the classical sense, the domination problem can be stated as

Copyright 2016 by the Tusi Mathematical Research Group.

Received Nov. 8, 2015; Accepted May 11, 2016.

${ }^{*}$ Corresponding author.

2010 Mathematics Subject Classification. Primary 46B42; Secondary $47 \mathrm{~B} 99$.

Keywords. narrow operators, dominated operators, lattice-normed spaces, Banach lattices. 
Ann. Funct. Anal. 7 (2016), no. 4, 646-655

http://dx.doi.org/10.1215/20088752-3660990

ISSN: 2008-8752 (electronic)

ANNALS of

FUNCTIONAL

http://projecteuclid.org/afa

ANALYSIS

\title{
DOMINATED OPERATORS FROM LATTICE-NORMED SPACES TO SEQUENCE BANACH LATTICES
}

\author{
NARIMAN ABASOV,${ }^{1}$ ABD EL MONEM MEGAHED,${ }^{2}$ and MARAT PLIEV ${ }^{3 *}$ \\ Communicated by M. de Jeu
}

\begin{abstract}
We show that every dominated linear operator from a BanachKantorovich space over an atomless Dedekind-complete vector lattice to a sequence Banach lattice $\ell_{p}(\Gamma)$ or $c_{0}(\Gamma)$ is narrow. As a consequence, we obtain that an atomless Banach lattice cannot have a finite-dimensional decomposition of a certain kind. Finally, we show that the order-narrowness of a linear dominated operator $T$ from a lattice-normed space $V$ to the Banach space with a mixed norm $(W, F)$ over an order-continuous Banach lattice $F$ implies the order-narrowness of its exact dominant $|T|$.
\end{abstract}

\section{INTRODUCTION AND PRELIMINARIES}

Narrow operators generalize compact operators defined on function spaces (see [11] for the first systematic study; see also the recent monograph [12]). Different classes of narrow operators in framework of vector lattices and latticenormed spaces were considered in [9], [10]. In the present article, we continue the investigation of narrow operators in lattice-normed spaces and show that every dominated linear operator from a Banach-Kantorovich space over an atomless Dedekind-complete vector lattice to a sequence Banach lattice is narrow. As a consequence, we obtain that an atomless Banach lattice cannot have a finitedimensional decomposition of a certain kind.

We also consider a domination problem for the exact dominant of a dominated linear operator. In the classical sense, the domination problem can be stated as

Copyright 2016 by the Tusi Mathematical Research Group.

Received Nov. 8, 2015; Accepted May 11, 2016.

${ }^{*}$ Corresponding author.

2010 Mathematics Subject Classification. Primary 46B42; Secondary $47 \mathrm{~B} 99$.

Keywords. narrow operators, dominated operators, lattice-normed spaces, Banach lattices. 
Ann. Funct. Anal. 7 (2016), no. 4, 646-655

http://dx.doi.org/10.1215/20088752-3660990

ISSN: 2008-8752 (electronic)

ANNALS of

FUNCTIONAL

http://projecteuclid.org/afa

ANALYSIS

\title{
DOMINATED OPERATORS FROM LATTICE-NORMED SPACES TO SEQUENCE BANACH LATTICES
}

\author{
NARIMAN ABASOV,${ }^{1}$ ABD EL MONEM MEGAHED,${ }^{2}$ and MARAT PLIEV ${ }^{3 *}$ \\ Communicated by M. de Jeu
}

\begin{abstract}
We show that every dominated linear operator from a BanachKantorovich space over an atomless Dedekind-complete vector lattice to a sequence Banach lattice $\ell_{p}(\Gamma)$ or $c_{0}(\Gamma)$ is narrow. As a consequence, we obtain that an atomless Banach lattice cannot have a finite-dimensional decomposition of a certain kind. Finally, we show that the order-narrowness of a linear dominated operator $T$ from a lattice-normed space $V$ to the Banach space with a mixed norm $(W, F)$ over an order-continuous Banach lattice $F$ implies the order-narrowness of its exact dominant $|T|$.
\end{abstract}

\section{INTRODUCTION AND PRELIMINARIES}

Narrow operators generalize compact operators defined on function spaces (see [11] for the first systematic study; see also the recent monograph [12]). Different classes of narrow operators in framework of vector lattices and latticenormed spaces were considered in [9], [10]. In the present article, we continue the investigation of narrow operators in lattice-normed spaces and show that every dominated linear operator from a Banach-Kantorovich space over an atomless Dedekind-complete vector lattice to a sequence Banach lattice is narrow. As a consequence, we obtain that an atomless Banach lattice cannot have a finitedimensional decomposition of a certain kind.

We also consider a domination problem for the exact dominant of a dominated linear operator. In the classical sense, the domination problem can be stated as

Copyright 2016 by the Tusi Mathematical Research Group.

Received Nov. 8, 2015; Accepted May 11, 2016.

${ }^{*}$ Corresponding author.

2010 Mathematics Subject Classification. Primary 46B42; Secondary $47 \mathrm{~B} 99$.

Keywords. narrow operators, dominated operators, lattice-normed spaces, Banach lattices. 
Ann. Funct. Anal. 7 (2016), no. 4, 646-655

http://dx.doi.org/10.1215/20088752-3660990

ISSN: 2008-8752 (electronic)

ANNALS of

FUNCTIONAL

http://projecteuclid.org/afa

ANALYSIS

\title{
DOMINATED OPERATORS FROM LATTICE-NORMED SPACES TO SEQUENCE BANACH LATTICES
}

\author{
NARIMAN ABASOV,${ }^{1}$ ABD EL MONEM MEGAHED,${ }^{2}$ and MARAT PLIEV ${ }^{3 *}$ \\ Communicated by M. de Jeu
}

\begin{abstract}
We show that every dominated linear operator from a BanachKantorovich space over an atomless Dedekind-complete vector lattice to a sequence Banach lattice $\ell_{p}(\Gamma)$ or $c_{0}(\Gamma)$ is narrow. As a consequence, we obtain that an atomless Banach lattice cannot have a finite-dimensional decomposition of a certain kind. Finally, we show that the order-narrowness of a linear dominated operator $T$ from a lattice-normed space $V$ to the Banach space with a mixed norm $(W, F)$ over an order-continuous Banach lattice $F$ implies the order-narrowness of its exact dominant $|T|$.
\end{abstract}

\section{INTRODUCTION AND PRELIMINARIES}

Narrow operators generalize compact operators defined on function spaces (see [11] for the first systematic study; see also the recent monograph [12]). Different classes of narrow operators in framework of vector lattices and latticenormed spaces were considered in [9], [10]. In the present article, we continue the investigation of narrow operators in lattice-normed spaces and show that every dominated linear operator from a Banach-Kantorovich space over an atomless Dedekind-complete vector lattice to a sequence Banach lattice is narrow. As a consequence, we obtain that an atomless Banach lattice cannot have a finitedimensional decomposition of a certain kind.

We also consider a domination problem for the exact dominant of a dominated linear operator. In the classical sense, the domination problem can be stated as

Copyright 2016 by the Tusi Mathematical Research Group.

Received Nov. 8, 2015; Accepted May 11, 2016.

${ }^{*}$ Corresponding author.

2010 Mathematics Subject Classification. Primary 46B42; Secondary $47 \mathrm{~B} 99$.

Keywords. narrow operators, dominated operators, lattice-normed spaces, Banach lattices. 
Ann. Funct. Anal. 7 (2016), no. 4, 646-655

http://dx.doi.org/10.1215/20088752-3660990

ISSN: 2008-8752 (electronic)

ANNALS of

FUNCTIONAL

http://projecteuclid.org/afa

ANALYSIS

\title{
DOMINATED OPERATORS FROM LATTICE-NORMED SPACES TO SEQUENCE BANACH LATTICES
}

\author{
NARIMAN ABASOV,${ }^{1}$ ABD EL MONEM MEGAHED,${ }^{2}$ and MARAT PLIEV ${ }^{3 *}$ \\ Communicated by M. de Jeu
}

\begin{abstract}
We show that every dominated linear operator from a BanachKantorovich space over an atomless Dedekind-complete vector lattice to a sequence Banach lattice $\ell_{p}(\Gamma)$ or $c_{0}(\Gamma)$ is narrow. As a consequence, we obtain that an atomless Banach lattice cannot have a finite-dimensional decomposition of a certain kind. Finally, we show that the order-narrowness of a linear dominated operator $T$ from a lattice-normed space $V$ to the Banach space with a mixed norm $(W, F)$ over an order-continuous Banach lattice $F$ implies the order-narrowness of its exact dominant $|T|$.
\end{abstract}

\section{INTRODUCTION AND PRELIMINARIES}

Narrow operators generalize compact operators defined on function spaces (see [11] for the first systematic study; see also the recent monograph [12]). Different classes of narrow operators in framework of vector lattices and latticenormed spaces were considered in [9], [10]. In the present article, we continue the investigation of narrow operators in lattice-normed spaces and show that every dominated linear operator from a Banach-Kantorovich space over an atomless Dedekind-complete vector lattice to a sequence Banach lattice is narrow. As a consequence, we obtain that an atomless Banach lattice cannot have a finitedimensional decomposition of a certain kind.

We also consider a domination problem for the exact dominant of a dominated linear operator. In the classical sense, the domination problem can be stated as

Copyright 2016 by the Tusi Mathematical Research Group.

Received Nov. 8, 2015; Accepted May 11, 2016.

${ }^{*}$ Corresponding author.

2010 Mathematics Subject Classification. Primary 46B42; Secondary $47 \mathrm{~B} 99$.

Keywords. narrow operators, dominated operators, lattice-normed spaces, Banach lattices. 
Ann. Funct. Anal. 7 (2016), no. 4, 646-655

http://dx.doi.org/10.1215/20088752-3660990

ISSN: 2008-8752 (electronic)

ANNALS of

FUNCTIONAL

http://projecteuclid.org/afa

ANALYSIS

\title{
DOMINATED OPERATORS FROM LATTICE-NORMED SPACES TO SEQUENCE BANACH LATTICES
}

\author{
NARIMAN ABASOV,${ }^{1}$ ABD EL MONEM MEGAHED,${ }^{2}$ and MARAT PLIEV ${ }^{3 *}$ \\ Communicated by M. de Jeu
}

\begin{abstract}
We show that every dominated linear operator from a BanachKantorovich space over an atomless Dedekind-complete vector lattice to a sequence Banach lattice $\ell_{p}(\Gamma)$ or $c_{0}(\Gamma)$ is narrow. As a consequence, we obtain that an atomless Banach lattice cannot have a finite-dimensional decomposition of a certain kind. Finally, we show that the order-narrowness of a linear dominated operator $T$ from a lattice-normed space $V$ to the Banach space with a mixed norm $(W, F)$ over an order-continuous Banach lattice $F$ implies the order-narrowness of its exact dominant $|T|$.
\end{abstract}

\section{INTRODUCTION AND PRELIMINARIES}

Narrow operators generalize compact operators defined on function spaces (see [11] for the first systematic study; see also the recent monograph [12]). Different classes of narrow operators in framework of vector lattices and latticenormed spaces were considered in [9], [10]. In the present article, we continue the investigation of narrow operators in lattice-normed spaces and show that every dominated linear operator from a Banach-Kantorovich space over an atomless Dedekind-complete vector lattice to a sequence Banach lattice is narrow. As a consequence, we obtain that an atomless Banach lattice cannot have a finitedimensional decomposition of a certain kind.

We also consider a domination problem for the exact dominant of a dominated linear operator. In the classical sense, the domination problem can be stated as

Copyright 2016 by the Tusi Mathematical Research Group.

Received Nov. 8, 2015; Accepted May 11, 2016.

${ }^{*}$ Corresponding author.

2010 Mathematics Subject Classification. Primary 46B42; Secondary $47 \mathrm{~B} 99$.

Keywords. narrow operators, dominated operators, lattice-normed spaces, Banach lattices. 